\title{
ARTÉRIA CEREBRAL MÉDIA HIPERDENSA EM ACIDENTE VASCULAR CEREBRAL: UM RELATO DE CASO
}

\section{ARTIGO ORIGINAL}

RODRIGUES, Jonathan Matheus Martins ', PEREIRA, Anne Caroline Castro 2, FRANÇA, Bruna Paiva de ${ }^{3}$, ALARCÃO, Eduarda Luz Barbosa ${ }^{4}$, RAMOS, Nathalia Moura ${ }^{5}$, COCENTINO, Luiz Matheus Xavier ${ }^{6}$

RODRIGUES, Jonathan Matheus Martins. Et al. Artéria cerebral média hiperdensa em acidente vascular cerebral: um relato de caso. Revista Científica Multidisciplinar Núcleo do Conhecimento. Ano. 06, Ed. 09, Vol. 06, pp. 39-50.

Setembro 2021. ISSN: 2448-0959, Link de acesso: https://www.nucleodoconhecimento.com.br/saude/arteria-cerebral, $\quad$ DOI: 10.32749/nucleodoconhecimento.com.br/saude/arteria-cerebral

\section{RESUMO}

A artéria cerebral média é um importante ramo da artéria carótida e sua oclusão resulta em acidente vascular cerebral isquêmico (AVCi). Em alguns casos, na tomografia computadorizada (TC) de crânio dessa condição é possível observar o sinal da artéria cerebral média hiperdensa (SACMH), uma imagem direta do trombo intravascular, em geral, na fase precoce de sua formação. Este estudo objetiva relatar um caso de AVCi com presença de SACMH na TC de crânio, pretendendo

${ }^{1}$ Graduação em Medicina. ORCID: 0000-0002-0121-3177

${ }^{2}$ Graduanda em Medicina. ORCID: 0000-0003-4570-1022

${ }^{3}$ Graduanda em Medicina. ORCID: 0000-0001-9619-4038

${ }^{4}$ Graduanda em Medicina. ORCID: 0000-0002-7054-4746

${ }^{5}$ Graduanda em Medicina. ORCID: 0000-0003-1033-4496

${ }^{6}$ Graduação em Medicina. ORCID: 0000-0002-9434-9865

$\mathrm{RC}: 97767$

Disponível em: https://www.nucleodoconhecimento.com.br/saude/arteria-cerebral 
apresentar a interpretação do achado desse sinal em uma tomografia e sua utilidade no diagnóstico e abordagem do paciente. Para isso, foram realizadas revisão do prontuário do caso e revisão da literatura nas bases de dados PubMed e Scielo, por meio dos descritores "Middle Cerebral Artery", "Hyperdense", "Stroke", "Computed Tomography" e "Neuroanatomy", no período de junho de 2021. Os achados demonstraram haver alta especificidade do SACMH no diagnóstico de AVCi, possibilitando que este seja precoce. Além disso, é eficiente na determinação de um prognóstico pior da patologia por ser associado a AVCs de maior volume. Por fim, é relevante sua relação com resistência à trombólise, indicando possíveis respostas ruins ao tratamento.

Descritores: Artéria Cerebral Média, Acidente Vascular Cerebral, Tomografia Computadorizada, Neuroanatomia.

\section{INTRODUÇÃO}

A artéria cerebral média ( $\mathrm{ACM})$, sendo o maior ramo terminal da artéria carótida interna e fazendo parte do polígono de Willis, possui extenso significado clínico. Divide-se em quatro segmentos cirúrgicos principais, denominados M1, M2, M3 e M4. Sua oclusão arterial impede a perfusão de sangue oxigenado para o parênquima cerebral, o que resulta em um acidente vascular cerebral (AVC) isquêmico, com consequente edema e necrose do tecido parênquima cerebral (NAVARRO-OROZCO e SANCHEZ-MANSO, 2020).

Em pacientes com AVC isquêmico agudo, o exame de tomografia computadorizada (TC) sem contraste é a modalidade básica de imagem. O procedimento é rápido, barato e amplamente disponível. Seus objetivos em pacientes com sintomas clínicos de AVC são procurar características precoces de AVC, excluir outras patologias intracranianas, como tumor, e excluir hemorragias que impedem a terapia trombolítica dentro da janela de tempo (CHRZAN et al., 2017).

RC: 97767

Disponível em: https://www.nucleodoconhecimento.com.br/saude/arteria-cerebral 
Além das anormalidades do parênquima cerebral na TC, em alguns pacientes é possível detectar um segmento hiperdenso de um vaso, o que representa imagem direta do trombo intravascular. Embora possa ser visto em qualquer vaso, é observado com maior frequência na ACM como o sinal da artéria cerebral média hiperdensa (SACMH) (CHRZAN et al., 2017).

O SACMH na TC de crânio é uma condição observada em $15 \%$ a $35 \%$ dos pacientes com AVC isquêmico (SUN et al., 2020). O SACMH foi identificado como trombos formados na fase aguda com um acúmulo de uma série de componentes sanguíneos, incluindo eritrócitos, fibrina e detritos celulares, que são indicativos de um trombo vermelho com maior resistência à trombólise (SUN et al., 2020). Estudos histopatológicos demonstram que sua composição pode ser dividida em fase precoce, com contagem de glóbulos vermelhos dominante ou glóbulos vermelhos na mesma proporção de fibrina, e fase tardia, com fibrina dominante. O SACMH é mais comumente visto na fase precoce (CHRZAN et al., 2017).

Quanto às apresentações clínicas, um infarto de grande ocupação territorial da ACM pode estar associado a edema cerebral massivo com aumento da pressão intracraniana (PIC) e ao aumento da morte celular isquêmica, com consequente hérnia cerebral e óbito. Associado com um pior prognóstico, foi descrito como infarto maligno de ACM (ALEXANDER et al., 2016; MOHAN et al., 2017; NEUGEBAUER et al., 2019). O SACMH, particularmente, está tipicamente associado ainda a um pior resultado clínico, com maior volume do AVC e déficit neurológico mais grave (CHRZAN et al., 2017).

As opções terapêuticas habituais para AVC agudo e edema grave consistem em reduzir a PIC utilizando hiperosmóticos, ventilação artificial e hiperventilação, hipotermia terapêutica, posição da cabeça elevada e sedativos (ALEXANDER et al., 2016). Os achados de metanálise de Saber et al (SABER et al., 2018) demonstraram resultados favoráveis à trombectomia mecânica para pacientes com oclusões M2 
que podem ser acessadas com segurança. $O$ tratamento cirúrgico, mais agressivo, reside na craniotomia descompressiva (CD) com o objetivo de reduzir a PIC (BONGIORNI et al., 2017). Conforme conclusão de estudo de Bongiorni et al. (2017), quando realizada com base em protocolo institucional rigoroso, a CD é uma ótima conduta para a diminuição das taxas de morbimortalidade.

O presente estudo tem como objetivo relatar o caso de um paciente com acidente vascular cerebral isquêmico com presença de hiperdensidade da artéria cerebral média esquerda na TC de crânio. Pretende-se através deste apresentar a interpretação do achado do sinal da artéria cerebral média hiperdensa em uma tomografia computadorizada de crânio e indicar sua utilidade no diagnóstico e abordagem do paciente.

As informações contidas neste trabalho foram obtidas por meio de revisão do prontuário do paciente, bem como por revisão da literatura de artigos publicados no período de 2011 a 2021 na base de dados PubMed e Scielo, por meio da utilização dos descritores "Middle Cerebral Artery", "Hyperdense", "Stroke", "Computed Tomography" e "Neuroanatomy". A coleta de dados foi realizada no período entre 18/06/2021 e 21/06/2021. Como critérios de inclusão, foram usados artigos que tinham como assunto principal o sinal da artéria cerebral média em acidentes vasculares encefálicos e a neuroanatomia local dentro da base de dados. Foram excluídos artigos anteriores ao ano de 2011 e que não abordassem o tema. Os estudos encontrados foram submetidos à análise crítica, dos quais foram selecionados 19 artigos. 


\section{RELATO DE CASO}

\subsection{ANAMNESE}

F.C.S, masculino, 68 anos, admitido no Pronto-Socorro do Hospital Público, em Brasília-DF, com quadro de hemiplegia à direita, em todo o hemicorpo iniciado 7 horas antes. O paciente foi internado na sala vermelha em grave estado geral, com Escala de Glasgow de 13 pontos, estável hemodinamicamente sem uso de drogas vasoativas com eliminações preservadas. A história patológica prévia do paciente incluía hipertensão arterial sistêmica, diabetes mellitus tipo 2, doença arterial coronariana e hiperplasia prostática benigna, sendo seus medicamentos de uso contínuo Metoprolol 50mg, Furosemida 40mg, Metformina 850mg, AAS 100mg, insulina NPH e sinvastatina.

\subsection{EXAME FÍSICO}

O paciente encontrava-se em grave estado geral, agitado, contactuante, não verbalizante, normocorado, hidratado, acianótico e anictérico, com pressão arterial de 160 x $80 \mathrm{mmHg}$, frequência cardíaca de 91 batimentos por minuto e saturando 95\% em uso de máscara não reinalante. O aparelho respiratório apresentava murmúrios vesiculares fisiológicos sem ruídos adventícios; o aparelho cardiovascular estava com ritmo cardíaco regular, em 2 tempos, com bulhas normofonéticas e sem sopros; o abdome estava plano e flácido, com ruídos hidroaéreos presentes, percussão timpânica e indolor à palpação; e ao exame neurológico pontuava 13 na Escala de Glasgow, apresentava pupilas isocóricas fotorreagentes, reflexo fotomotor preservado e aparente hemiplegia. As extremidades tinham pulsos palpáveis, amplos, cheios e sem edemas. 


\subsection{EXAMES SUBSIDIÁRIOS}

Os exames laboratoriais apresentaram glicemia de $430 \mathrm{mg} / \mathrm{dL}$, potássio $5.32 \mathrm{mEq} / \mathrm{L}$, sódio $134.4 \mathrm{mEq} / \mathrm{L}$, uréia $53.2 \mathrm{mg} / \mathrm{dL}$, creatinina 1.45 , hemácias $4.21 \times 10^{3} / \mathrm{mm}^{3}$, VCM $100.5 \mathrm{fL}$, leucócitos $7.9 \times 10^{3} / \mathrm{mm}^{3}$, neutrófilos $88,4 \%$, linfócitos $7.2 \times 10^{3} / \mathrm{mm}^{3}$, plaquetas 275 e tempo de protrombina 14,2 segundos. A tomografia de crânio, segundo laudo de departamento de radiologia, apresentou extensa área de hipodensidade com perda da diferenciação entre substância branca e cinzenta, acometendo os lobos frontal, parietal, temporal e insular esquerdos, em território vascular da artéria cerebral média esquerda, sem efeito expansivo ou atrófico significativo, sugerindo isquemia aguda. Além disso, demonstrou hiperdensidade da artéria cerebral média esquerda, sinais de redução volumétrica encefálica com acentuação em sulcos, fissuras e cisternas cerebrais com dilatação compensatória do sistema ventricular. Havia focos de hipodensidade na substância branca profunda e periventricular supratentorial e ateromatose carotídea.

\subsection{HIPÓTESE DIAGNÓSTICA}

Feita a hipótese diagnóstica de acidente vascular cerebral isquêmico em hemisfério esquerdo.

\subsection{CONDUTA}

Nesse contexto foi iniciado protocolo institucional para AVCi fora de janela e após estabilização do paciente ele foi encaminhado para unidade de terapia intensiva.

\section{DISCUSSÃO}

A TC de crânio fornece informações úteis sobre a composição e a quantidade de coágulos, especialmente em casos como o AVC de ACM. A presença e o comprimento do $\mathrm{SACMH}$, indicando trombose de segmentos M1 e M2 proximal, RC: 97767

Disponível em: https://www.nucleodoconhecimento.com.br/saude/arteria-cerebral 
fornecem informações diagnósticas e prognósticas. No entanto, sua utilidade clínica é aparentemente subutilizada (TOPCUOGLU et al., 2015). Diante disso, é muito importante que os radiologistas reconheçam esse sinal, uma vez que ajuda a salvar o paciente na janela de tempo para a trombólise (CHRZAN et al., 2017).

De acordo com pesquisa de Topcuoglu et al (2015), os exames de TC sem contraste apresentaram SACMH em 2 de cada 5 pacientes com oclusão aguda de ACM. A incidência relatada de artéria hiperdensa mostra variabilidade significativa, que é em grande parte por causa do tempo para a varredura e da espessura do corte usado, sendo uma das dificuldades para a detecção do SACMH (TOPCUOGLU et al., 2015; CHRZAN et al., 2017). A grande espessura de corte, atingindo $5 \mathrm{~mm}$, em comparação com o diâmetro de ACM de 2-3 mm, pode resultar em efeitos de volume parcial borrando a hiperdensidade intraluminal e reduzindo a sensibilidade de detecção do SACMH. Portanto, sugere-se o uso de cortes finos de cerca de $1 \mathrm{~mm}$ para a avaliação de exames de TC sem contraste para aumentar a sensibilidade (CHRZAN et al., 2017).

O SACMH é visto na TC como uma hiperatenuação focal ao nível da ACM em comparação a artéria contralateral devido a maior quantidade de proteína e fibrina e menor proporção de soro, e é um dos primeiros sinais radiológicos do AVC isquêmico agudo, podendo ser observado em 90 minutos após o início do quadro (CHIENG et al., 2020; MEZA e CORREA, 2020). Pode ocorrer de maneira unilateral, relacionada principalmente a doença tromboembólica, ou bilateral, associada à disfunção fisiológica subjacente ou como artefato por calcificação vascular ou hipodensidade parenquimatosa próxima (VOGLER et al., 2018). Devido à sua visibilidade precoce após a oclusão do vaso e antes das alterações isquêmicas parenquimatosas patológicas, o SACMH auxilia no diagnóstico de AVC agudo em tempo crítico (CHIENG et al., 2020). 
A localização da hiperdensidade na TC simples não especifica o local da oclusão arterial (TOPCUOGLU et al., 2015). De acordo com resultados de Chrzan et al. (2017), o segmento hiperdenso longo da ACM e o acometimento da parte proximal ou distal do vaso são associados a um desfecho significativamente pior, no entanto, o segmento hiperdenso curto da ACM não exclui grande área de danos no tecido cerebral (CHRZAN et al., 2017).

Este sinal é considerado um indicador de alta especificidade para AVC isquêmico agudo, cerca de $95 \%$ a $100 \%$, porém de sensibilidade relativamente baixa, que pode variar de 37\% a 54\% (CHIENG et al., 2020; REMON et al., 2020). Podem ocorrer falsos-positivos, principalmente nos casos de calcificação parietais, especialmente nos segmentos médio e distal dos segmentos M1 das ACMs, ou nos casos de hemorragias subaracnóideas focais (GONÇALVES et al., 2011). Observa-se ainda maior taxa de falsos-positivos nos acometimentos bilaterais e nos pacientes com hematócrito elevado, doença arteriosclerótica ou naqueles sem sintomatologia com desidratação ou poliglobulia (MEZA e CORREA, 2020).

Assim, é importante atentar-se a diagnósticos diferenciais na presença da hiperdensidade da ACM associada a achados como lipo-hialinose, depósitos de lipídeos, calcificação, presença de contraste intravenosa, hematócrito elevado, infecção e contusão ou tumores adjacentes, considerando que, nestes casos, o achado da hiperdensidade não necessariamente corresponde ao SACMH (REMON et al., 2020).

A apresentação clínica do AVC varia conforme o sítio acometido, comumente havendo déficits focais duradouros (> 24 horas) e rebaixamento do nível de consciência (OLIVEIRA, 2014). O SACMH está tipicamente associado a AVCs de maior volume, geralmente envolvendo um infarto de pelo menos dois terços do território da $\mathrm{ACM}$, o que implica em maiores danos neurológicos e alta mortalidade nesses pacientes. Sabe-se, então, que a clínica do AVC com SACMH é 
caracterizada por edema extenso, que leva ao aumento da PIC e herniação (ALEXANDER et al., 2016; SUN et al., 2020; MOHAN RAJWANI et al., 2017; DAS et al., 2019; NEUGEBAUER et al., 2019).

Nesse contexto, em decorrência da elevação da PIC, esses pacientes apresentam hemiplegia e deterioração da consciência nas primeiras 48h após o AVC. Esse comprometimento pode se desenvolver em até $3 \mathrm{~h}$ do evento vascular, em decorrência do edema cerebral, que progride rapidamente de minutos a horas, podendo causar hérnia subfalcina, uncal, transtentorial e/ou tonsilar, e culminar, inclusive, em morte encefálica (ALEXANDER et al., 2016; MOHAN RAJWANI et al., 2017; DAS et al., 2019; NEUGEBAUER et al., 2019).

Ademais, a oclusão do tronco principal da ACM, por provocar infarto da maior parte do hemisfério ipsilateral, se manifesta com um quadro clássico de hemiparesia e hemiplegia de membros e face contralaterais, hemianopsia ou quadrantanopsia contralaterais, afasia no caso de acometimento do hemisfério esquerdo ou heminegligência e desorientação espacial quando acometido o direito. De maneira geral, as manifestações costumam se estabilizar após o quinto dia de evolução, fase máxima do edema cerebral, porém os déficits tendem a ser permanentes (OLIVEIRA, 2014).

Na revisão sistemática e metanálise de Sun et al. (2020), os pacientes com SACMH inicial apresentaram 1,56 vezes mais chance de ter desfecho desfavorável funcional do que os pacientes que não o apresentaram. Em primeiro lugar, o SACMH representa a oclusão da artéria cerebral média por um trombo grande, que pode provocar a formação de um grande infarto, que seja insensível à trombólise. Em segundo lugar, o SACMH está associado a um trombo saturado de eritrócitos, fibrina e detritos celulares, um conteúdo considerado resistente à trombólise utilizando tPA. 
O prognóstico de pacientes com infarto de ACM é determinado por fatores clínicos e radiológicos, como a extensão do comprimento hiperdenso da $A C M$, além da localização do trombo na parte proximal ou distal dela. Sabe-se que o seguimento hiperdenso longo está mais associado a piores prognósticos (CHRZAN et al., 2017; DAS et al., 2019). Segundo Shi (2021), a persistência do SACMH é um fator de prognóstico precoce de mau resultado funcional, em comparação ao seu desaparecimento, que está associado a taxas duas vezes maiores de bom resultado funcional. Quanto ao lado de acometimento da ACM, não deve ser utilizado como marcador prognóstico, considerando que não se observou influência na mortalidade dos pacientes em três meses (DUCCl et al., 2015).

Para o tratamento do AVC isquêmico, deve-se levar em consideração o tempo de evolução do episódio, considerado a partir do momento de instalação do quadro neurológico. De maneira geral, um intervalo $<4.5$ horas indica trombólise com tPA e um intervalo $>4.5$ horas ou desconhecido contraindica a trombólise (OLIVEIRA, 2014). Quanto aos pacientes com $S A C M H$, os dados quanto à terapêutica ideal ainda são limitados e as taxas e extensão de recuperação daqueles tratados com tPA são altamente variáveis (PALIWAL et al., 2012).

Independente da trombólise, sabe-se que a presença do SACMH na TC de admissão associa-se frequentemente a resultados ruins, o que pode ocorrer devido a maior carga de coágulos e AVC grave associado (PALIWAL et al., 2012). O estudo de Sun et al. (2020) demonstra que, mesmo restringindo a janela de tratamento há três horas, a presença inicial do sinal na TC de crânio ainda se associa a maiores taxas de desfechos desfavoráveis em comparação aos pacientes que também receberam trombólise, mas que não apresentavam SACMH no pré-tratamento.

Após a trombólise sistêmica, o sinal pode desaparecer, representando dissolução do coágulo, ou persistir na TC de crânio acompanhamento. De acordo com o estudo de Prakash et al, a persistência do achado da hiperdensidade da ACM no 
acompanhamento de pacientes com AVC isquêmico agudo tratados com tPA pode ser utilizada como um preditor precoce de mau resultado funcional (PALIWAL et al., 2012).

\section{CONSIDERAÇÕES FINAIS}

Este estudo teve como pretensão apresentar a interpretação do achado do sinal da artéria cerebral média hiperdensa em uma tomografia computadorizada de crânio e indicar sua utilidade no diagnóstico e abordagem do paciente.

Como interpretação do sinal da artéria cerebral média hiperdensa, tem-se que ele representa a imagem direta do trombo na TC e sua presença fornece informações diagnósticas e prognósticas relevantes, sendo um dos primeiros sinais radiológicos do AVC isquêmico agudo, com especificidade entre 95 e 100\% para tal patologia, o que possibilita seu diagnóstico precoce. Já em relação a sua utilidade, este sinal está associado a AVCs de maior volume, indicando mais danos neurológicos e pior prognóstico, estando relacionado a edema cerebral, aumento da PIC e herniação. Por ocorrer na presença de trombos grandes e com conteúdo resistente à trombólise, sua presença também indica uma possível resposta ruim ao tratamento, podendo o trombo e consequentemente o sinal desaparecer após o medicamento ou não, sendo uma indicação do prognóstico do paciente.

\section{REFERÊNCIAS}

ALEXANDER, Paul et al. Hemicraniectomy versus medical treatment with large MCA infarct: a review and meta-analysis. BMJ open, v. 6, n. 11, p. e014390, 2016. Disponível em: https://pubmed.ncbi.nlm.nih.gov/27884858/. Acesso em: 19/06/2021.

BONGIORNI, Gianise Toboliski et al. Decompressive craniotomy for the treatment of malignant infarction of the middle cerebral artery: mortality and outcome. Arquivos de neuropsiquiatria, v. 75 , p. 424-428, 2017. Disponível em:

RC: 97767

Disponível em: https://www.nucleodoconhecimento.com.br/saude/arteria-cerebral 
https://www.scielo.br/j/anp/a/w95864vzG3YYVwpyNWqPFyC/?lang=en. Acesso em: 20/06/2021.

CHIENG Julian Sau Lian et al. The hyperdense vessel sign in cerebral computed tomography: pearls and pitfalls. Singapore medical journal, v. 61 , n. 5 , p. 230 , 2020. Disponível em: https://pubmed.ncbi.nlm.nih.gov/32754771/. Acesso em 18/06/2021.

CHRZAN, Robert; GLEŃ, Agnieszka; URBANIK, Andrzej. Hyperdense middle cerebral artery sign as the only radiological manifestation of hyperacute ischemic stroke in computed tomography. Neurologia i neurochirurgia polska, v. 51, n. 1, p. 33-37, 2017. Disponível em: https://pubmed.ncbi.nlm.nih.gov/28341040/. Acesso em: 18/06/2021.

DAS Suparna et al. Decompressive hemicraniectomy in the treatment of malignant middle cerebral artery infarction: a meta-analysis. World neurosurgery, v. 123, p. 816, 2019. Disponível em: https://pubmed.ncbi.nlm.nih.gov/30500591/. Acesso em 21/06/2021.

DUCCI, Renata Dal-Prá et al. Does the side of middle cerebral artery compromise matters in the mortality after thrombolysis in ischemic stroke? Arquivos de neuropsiquiatria, v. 73 , p. 644-647, 2015. Disponível em: https://pubmed.ncbi.nlm.nih.gov/26222353/. Acesso em 18/06/2021.

GONÇALVES, Fabrício Guimarães et al. Sinais em neurorradiologia: parte 1. Radiologia Brasileira, v. 44, p. 123-128, 2011. Disponível em: https://www.scielo.br/j/rb/a/L5NL4PSHJjFHv3JKT7rjrDz/?lang=pt. Acesso em 20/06/2021.

MEZA, Alexander Roman; CORREA, Celso Huaman. Radiological semiology in emergency brain pathology. Revista de La Facultad de Medicina Humana, Lima, 
v. 20, n. 1, p. 130-137, 2020. Disponível em: http://www.scielo.org.pe/pdf/rfmh/v20n1/en_2308-0531-rfmh-20-01-130.pdf. Acesso em 18/06/2021.

MOHAN RAJWANI, Kapil; CROCKER, Matthew; MOYNIHAN, Barry. Decompressive craniectomy for the treatment of malignant middle cerebral artery infarction. British journal of neurosurgery, v. 31, n. 4, p. 401-409, 2017. Disponível em: https://pubmed.ncbi.nlm.nih.gov/28604106/. Acesso em: 21/06/2021.

NAVARRO-OROZCO, Daniel; SÁNCHEZ-MANSO, Juan Carlos. Neuroanatomy, middle cerebral artery. StatPearls [Internet], 2020. Disponível em: https://pubmed.ncbi.nlm.nih.gov/30252258/. Acesso em: 21/06/2021.

NEUGEBAUER, Hermann et al. Outcomes of hypothermia in addition to decompressive hemicraniectomy in treatment of malignant middle cerebral artery stroke: a randomized clinical trial. JAMA neurology, v. 76, n. 5, p. 571-579, 2019. Disponível em: https://pubmed.ncbi.nlm.nih.gov/30657812/. Acesso em: 18/06/2021.

OLIVEIRA, Marcelo Magaldi Ribeiro de. ANÁLISE DAS IMAGENS. Rev Med Minas Gerais, v. 24, n. 4, p. 560-563, 2014. Disponível em: http://www.rmmg.org/sumario/139. Acesso em: 19/06/2021.

PALIWAL, Prakash R. et al. Persistence of hyperdense middle cerebral artery sign on follow-up CT scan after intravenous thrombolysis is associated with poor outcome. Cerebrovascular Diseases, v. 33, n. 5, p. 446-452, 2012.Disponível em: https://pubmed.ncbi.nlm.nih.gov/22456065/. Acesso em 20/06/2021.

REMON, Ariel Sosa; ALVAREZ, Ana Esperanza Jerez; CHAVEZ, Carmen Esther Remon; Signo de la arteria cerebral média hiperdensa e ictus isquémico con transformación hemorrágica. Revista Finlay, v. 10, n. 4, p. 440-444, 2020. 
Disponível em: http://scielo.sld.cu/scielo.php?script=sci_arttext\&pid=S2221 2434202000040044. Acesso em 19/06/2021.

SABER, Hamidreza et al. Mechanical thrombectomy for acute ischemic stroke with occlusion of the M2 segment of the middle cerebral artery: a meta-analysis. Journal of neurointerventional surgery, v. 10, n. 7, p. 620-624, 2018. Disponível em: https://pubmed.ncbi.nlm.nih.gov/29127196/. Acesso em: 18/06/2021.

SHI, Chenyu; KILLINGSWORTH, Murray C.; BHASKAR, Sonu Menachem Maimonides. Prognostic capacity of hyperdense middle cerebral artery sign in anterior circulation acute ischaemic stroke patients receiving reperfusion therapy: a systematic review and meta-analysis. Acta Neurologica Belgica, p. 1-13, 2021. Disponível em: https://pubmed.ncbi.nlm.nih.gov/34095978/. Acesso em: 19/06/2021.

SUN, Huanhuan et al. Intravenous thrombolysis for ischemic stroke with hyperdense middle cerebral artery sign: a meta-analysis. Acta Neurologica Scandinavica, v. 141, n. 3, p. 193-201, 2020. Disponível em: https://pubmed.ncbi.nlm.nih.gov/31598961/. Acesso em: 20/06/2021.

TOPCUOGLU, Mehmet A.; ARSAVA, Ethem Murat; AKPINAR, Erhan. Clot characteristics on computed tomography and response to thrombolysis in acute middle cerebral artery stroke. Journal of Stroke and Cerebrovascular Diseases, v. 24, n. 6, p. 1363-1372, 2015. Disponível em: https://pubmed.ncbi.nlm.nih.gov/25804568/. Acesso em: 19/06/2021.

VOGLER, James et al. Bilateral hyperdense middle cerebral arteries: Stroke sign or not? Radiology case reports, v. 13, n. 5, p. 933-935, 2018. Disponível em: https://www.ncbi.nlm.nih.gov/pmc/articles/PMC6072911/. Acesso em 19/06/2021.

Enviado: Agosto, 2021.

RC: 97767

Disponível em: https://www.nucleodoconhecimento.com.br/saude/arteria-cerebral 
Aprovado: Setembro, 2021. 